\title{
Exactitud diagnóstica de la tomografía computarizada en la estadificación del cáncer de colon
}

\author{
Leonardo Cárcamo G. ${ }^{1}$, Héctor Henríquez L. ${ }^{2}$, Daniela Figueroa P. ${ }^{2}$, Felipe Imigo G. ${ }^{1}$, \\ Rodrigo San Martín B. ${ }^{2}$, María Elena Molina P. ${ }^{1}$ y Gonzalo Urrejola S. ${ }^{1}$
}

'Departamento de Cirugía Digestiva, Pontificia Universidad Católica de Chile. 2Departamento de Radiología, Pontificia Universidad Católica de Chile. Santiago, Chile.

Recibido 2020-11-18, aceptado 2021-01-21

Correspondencia a: Dr. Gonzalo Urrejola S. giurrejola@uc.cl

\section{Diagnostic accuracy of computed tomography in colon cancer staging}

Introduction: Neoadjuvant therapy is an emerging treatment for selected cases of colon cancer. The decision is based on the analysis of preoperative images taken by computed tomography. However, its ability to discriminate those who could benefit from this therapy is unknown. Aim: Our objective is to determine the diagnostic accuracy of computed tomography to evaluate the colon cancer stage and its correlation among expert radiologists. Materials and Method: Diagnostic accuracy study. After sample size calculation, we randomly selected 47 patients who underwent a colonic adenocarcinoma resection with curative intent and had available images up to 60 days after surgery. The depth of tumor and lymph nodal invasion were evaluated by two expert radiologists, blinded to each other. The differences between radiologists were audited by a third one. The results were correlated with biopsy as the gold standard. We calculated sensitivity, specificity, negative predictive value, positive predictive value, and diagnostic accuracy of computed tomography. The agreement between radiologists was calculated using the kappa index. Results: The accuracy to differentiate T3-T4 tumors from T1-T2 was $89.4 \%$. In contrast, ability to differentiate T4 tumors from T3 tumors was $65.9 \%$. The accuracy to determine an $\mathrm{N}(+)$ was $66 \%$. Agreement between radiologists was 0.929 to identify T1-2, 0.602 for T3, 0.584 for T4 tumors. To determine an $\mathrm{N}(+)$, the concordance was 0.521 . Conclusion: Diagnostic computed tomography accuracy in colon cancer is adequate to differentiate advanced tumors. However, its ability to discriminate between $\mathrm{T} 3$ and $\mathrm{T} 4, \mathrm{~N}(+)$ and the low agreement between radiologists suggests the need for complementary imaging techniques.

Keywords: colon cancer; computed tomography; neoadjuvant therapy; diagnostic accuracy.

\section{Resumen}

Introducción: Una terapia emergente para el cáncer de colon es la neoadyuvancia, en casos seleccionados. Dicha selección se basa en el análisis preoperatorio de imágenes mediante tomografía computada. Objetivo: Nuestro objetivo es determinar la exactitud diagnóstica del examen para estadificar el cáncer de colon y su correlación entre radiólogos expertos. Materiales y Método: Estudio de exactitud diagnóstica. Previo cálculo muestral se seleccionaron aleatoriamente 47 pacientes con adenocarcinoma colónico resecado con intención curativa e imágenes hasta 60 días previo cirugía. Se evaluó profundidad de invasión tumoral e invasión linfonodal por 2 radiólogos expertos y ciegos entre sí. Las diferencias fueron auditadas por un tercer radiólogo experto. Se compararon resultados con la biopsia, calculando la sensibilidad, especificidad, valor predictivo negativo, valor predictivo positivo y exactitud diagnóstica del examen. Se calculó la concordancia entre radiólogos mediante el índice de kappa. Resultados: La exactitud para diferenciar tumores T3-T4 de T1-T2 fue del 89,4\%. En cambio, la capacidad para diferenciar tumores T4 de los T3 fue de un $65,9 \%$. La exactitud para determinar un $\mathrm{N}(+)$ fue de un $66 \%$. La concordancia entre radiólogos fue de 0,929 para identificar tumores T1-2, de 0,602 para T3, de 0,584 para T4. Para determinar un N (+), la concordancia fue de 0,521 . Conclusión: La exactitud diagnóstica de la tomografía computada en cáncer de colon es adecuada para distinguir tumores avanzados. Sin embargo, su capacidad para discriminar entre $\mathrm{T} 3$ y T4, los $\mathrm{N}(+)$ y la baja concordancia entre radiólogos, sugiere la necesidad de buscar técnicas imagenológicas complementarias.

Palabras clave: cáncer de colon; tomografía computada; neoadyuvancia; exactitud diagnóstica. 


\section{Introducción}

El cáncer colorrectal es el tercero en términos de incidencia a nivel mundial y el segundo en nuestro país, tanto en incidencia como en mortalidad para ambos sexos ${ }^{1}$. Su tratamiento ha evolucionado del punto de vista quirúrgico y de las terapias oncológicas asociadas, logrando obtener sobrevidas globales y libres de enfermedad cada vez mejores ${ }^{2,3}$. El tratamiento estándar de esta patología consiste en la resección del segmento afectado por el tumor, seguido de quimioterapia adyuvante en caso de etapas II de alto riesgo y III ${ }^{4,5}$.

Con el objetivo de optimizar aún más los resultados oncológicos del tratamiento de estos tumores, se ha planteado entregar la quimioterapia antes del procedimiento quirúrgico en casos seleccionados (neoadyuvancia). El tratamiento neoadyuvante es ampliamente utilizado en cáncer de recto, estómago y esófago, donde ha demostrado beneficios oncológicos. En cáncer de colon (CC) no se efectúa de manera rutinaria, sin embargo, existe un creciente interés en su implementación ${ }^{6-8}$.

Los beneficios teóricos de la quimioterapia neoadyuvante son múltiples: pudiese ser más efectiva para erradicar las micrometástasis; mejorar la adherencia al tratamiento en casos seleccionados, ya que se independiza de las complicaciones posoperatorias; y además disminuir potencialmente el volumen tumoral. En su conjunto, esto ayudaría a controlar la diseminación de células tumorales durante la disección, mejorar la tasa de resecciones completas y permitir cirugías mínimamente invasivas con un menor índice de conversión? Es importante definir qué pacientes que son de alto riesgo oncológico y por lo tanto tendrían un mayor beneficio de recibir neoadyuvancia. La quimioterapia se asocia a morbilidad, costo y tiempo, por lo que debe evitarse en los pacientes que no obtendrían un beneficio categórico. La selección de dichos pacientes, considerados de alto riesgo, podría sustentarse en los hallazgos imagenológicos de la tomografía computarizada (TC).

La TC es la modalidad de imagen de elección para la estadificación del CC. Según un metaanálisis reciente, su sensibilidad y especificidad para identificar tumores avanzados, es decir T3 y $\mathrm{T} 4$, es de un $90 \%$ y $69 \%$, mientras que para el compromiso linfoganglionar es de un $71 \%$ y $67 \%$ respectivamente ${ }^{10}$.

El objetivo del presente estudio es determinar la exactitud diagnóstica (EXD) de la TC para estadificar el CC, su capacidad para discriminar tumores T4 de entre los tumores avanzados y analizar la concordancia entre radiólogos expertos al estadificar clínicamente el CC.

\section{Materiales y Método}

Este estudio de exactitud diagnóstica fue aprobado por el comité de ética institucional, autorizándose la dispensa del consentimiento informado. Se determinó un tamaño muestral mínimo de 45 pacientes con el fin de evaluar concordancia y exactitud diagnóstica. Los casos se recolectaron del registro electrónico de cáncer de colon, mantenido de forma prospectiva. La selección se realizó de manera aleatoria mediante software estadístico.

Se incluyó pacientes mayores de 18 años, con adenocarcinoma colónico resecado electivamente con intención curativa y TC disponible hasta 60 días previos a la cirugía. Se excluyeron pacientes operados de urgencia, resecciones no oncológicas, aquellos cuya indicación de resección fuera por un adenocarcinoma circunscrito a un pólipo, antecedente de neoadyuvancia y aquellos portadores de enfermedades hereditarias colorrectales.

\section{Especificaciones TC}

Las TC fueron realizadas con un scanner de 64 cortes (PHILIPS 64-slice Brilliance ${ }^{\circledR}$, MDCT, Philips, Netherlands) configurado con los siguientes parámetros: tiempo de rotación $0,75 \mathrm{~s}$, tono 1,2 y colimación 64 x 0,625 mm. Las imágenes con contraste fueron guardadas 70 segundos después de la inyección de contraste. El contraste utilizado fue $100 \mathrm{ml}$ de ioversol $68 \% 320 \mathrm{mg} / \mathrm{ml}$ a una velocidad de inyección de $3 \mathrm{ml} / \mathrm{s}$. Las imágenes fueron analizadas utilizando IMPAX (GE Healthcare, Barrington, IL, USA).

\section{Evaluación radiológica}

La evaluación de la profundidad de invasión tumoral (T) y la presencia de invasión linfonodal (N) se realizó mediante la octava edición de la clasificación $\mathrm{TNM}^{11}$. Las TC fueron evaluadas específicamente para el presente estudio, por dos radiólogos especialistas en patología digestiva, ciegos entre ellos y con el investigador principal. Las diferencias entre ellos fueron auditadas por un tercer radiólogo experto previo al cálculo de exactitud diagnóstica. Se clasificó el $\mathrm{T}$ en 3 categorías: T1/T2, T3 y T4. El N se clasificó en positivo o negativo. A cada radiólogo se le entregó la misma pauta donde debían marcar la alternativa que consideraban más adecuada ante el caso que se encontraban evaluando. 


\section{Análisis estadístico}

Se consideró como estándar de referencia a la biopsia de la pieza quirúrgica evaluada por patólogos expertos en cirugía colorrectal. Una vez auditadas las diferencias por el tercer radiólogo experto, se calculó la sensibilidad (S), especificidad (E), valor predictivo positivo (VPP), valor predictivo negativo (VPN) y EXD del informe radiológico comparado al

Tabla 1. Características histopatológicas de la serie

\begin{tabular}{|cr|}
\hline Variable & n $(\mathbf{\%})$ \\
\hline $\mathrm{pT}$ & $4(8,5 \%)$ \\
1 & $2(4,3 \%)$ \\
2 & $23(48,9 \%)$ \\
3 & $18(38,3 \%)$ \\
4 & \\
$\mathrm{pN}$ & $23(48,9 \%)$ \\
+ & $24(51,1 \%)$ \\
\hline
\end{tabular}

Tabla 2. Estadísticos para diferenciar T1-2 de entre todos los tumores

\begin{tabular}{|lccc|}
\hline & Radiólogo 1 & Radiólogo 2 & Auditado \\
\hline Sensibilidad & 83,3 & 83,3 & 83,3 \\
Especificidad & 90,2 & 92,7 & 90,2 \\
VPP & 55,6 & 62,5 & 55,6 \\
VPN & 97,4 & 97,4 & 97,4 \\
\hline
\end{tabular}

Tabla 3. Estadísticos para diferenciar T3 de entre todos los tumores

\begin{tabular}{|lccc|}
\hline & Radiólogo 1 & Radiólogo 2 & Auditado \\
Sensibilidad & 69,6 & 65,2 & 69,6 \\
Especificidad & 58,3 & 66,7 & 62,5 \\
VPP & 61,5 & 65,2 & 64,0 \\
VPN & 66,7 & 66,7 & 68,2 \\
\hline
\end{tabular}

Tabla 4. Estadísticos para diferenciar T4 de entre todos los tumores

\begin{tabular}{|lccc|}
\hline & Radiólogo 1 & Radiólogo 2 & Auditado \\
\hline Sensibilidad & 50,0 & 61,1 & 55,6 \\
Especificidad & 89,7 & 82,8 & 89,7 \\
VPP & 75,0 & 68,8 & 76,9 \\
VPN & 74,3 & 77,4 & 76,5 \\
\hline
\end{tabular}

estándar de referencia con un intervalo de confianza del 95\%. Se calculó la concordancia entre radiólogos utilizando el índice de kappa. Se consideró significancia estadística a un $\mathrm{p}<0,05$. Los datos fueron recolectados utilizando Microsoft Excel y analizados mediante SPSS v 22,0.

\section{Resultados}

Se seleccionaron aleatoriamente 47 casos que cumplieran con los criterios de inclusión y exclusión. La mayoría de los tumores fueron avanzados (T3-T4) y con distribución equitativa con respecto a los linfonodos comprometidos. Las características histopatológicas de los casos seleccionados se detallan en la Tabla 1.

La EXD calculada fue del $89,4 \%$ para diferenciar el grupo de tumores avanzados (T3-4) de los más iniciales (T1-2). La S y E fueron de $83,3 \%$ y 90,2\% respectivamente, con un VPN del 97,4\% (Tabla 2). Se sobre estadificaron $4(8,5 \%)$ pacientes y se infravaloró $1(2,12 \%)$ caso al realizar esta discriminación.

Los radiólogos pudieron diferenciar tumores T3 y T4 del resto de los tumores con una EXD del 66\% y $76,6 \%$ respectivamente. La S y E fueron del 69,6\% y $62,5 \%$ para identificar tumores T3. En cambio, para identificar tumores T4 se observó una $\mathrm{S}$ del $55,6 \%$ y una E del 89,7\% (Tablas 3 y 4).

En cuanto a la capacidad para discriminar los tumores T4 entre los tumores avanzados (T3-T4; $\mathrm{n}=41$ ), se obtuvo una EXD del $65,9 \%$, con una $\mathrm{S}$ del $55,6 \%$ y una E del $85 \%$. Los estadísticos separados por radiólogo se encuentran descritos en la Tabla 5 .

Con respecto al $\mathrm{N}$, se logró una valoración correcta en el $66 \%$ de los casos, donde en $9(19,1 \%)$ de ellos se sobre estadificó y en $7(14,9 \%)$ se infravaloró. En la Tabla 6 se puede observar cómo tanto los VPP y VPN bordean el 65\%.

La concordancia entre radiólogos presentó un índice de kappa de 0,929 para identificar tumores T1-2, de 0,602 para T3, de 0,584 para T4. La concordancia para identificar los pacientes con $\mathrm{N}(+)$ fue de 0,521 .

\section{Discusión}

Nuestro estudio demuestra que la TC, en el contexto de la estadificación preoperatoria del CC, presenta limitaciones a pesar de que sea analizada por radiólogos expertos. Nuestros resultados 
ARTÍCULO ORIGINAL

indican que se logra diferenciar correctamente a los tumores avanzados de los más precoces, presentando una EXD del $89,4 \%$ y una alta correlación entre radiólogos para ello. Sin embargo, la capacidad para diferenciar tumores T4 de T3, así como para identificar compromiso ganglionar es menor y presenta una importante variabilidad entre radiólogos.

Experiencias recientes se condicen con nuestros resultados y dan una señal de cautela ante la información entregada por la TC en CC. En la publicación de Friis et al. ${ }^{12}$, identificaron una sensibilidad y especificidad del $61 \%$ y $85 \%$ respectivamente para enfermedad localmente avanzada e incluso expresan que el $58 \%$ de los pacientes en estadio I fueron mal clasificados. En la publicación de Korsbakke et al. ${ }^{13}$, la concordancia entre radiólogos fue del 0,27 para el $\mathrm{T}$ y del 0,21 para el $\mathrm{N}$, inferiores a nuestros resultados.

La importancia de discriminar la profundidad de invasión entre los tumores avanzados radica en la necesidad de una adecuada selección. Una buena selección permitiría individualizar el tratamiento de los pacientes y conocer específicamente quiénes se beneficiarían con quimioterapia de manera preoperatoria ${ }^{9}$. Si se infravalora la profundidad de invasión tumoral se estaría privando a los pacientes de los potenciales beneficios de la neoadyuvancia. Por el contrario, una sobreestadificación, podría implicar tratamientos que conllevan morbilidad relevante, sin un beneficio categórico para el paciente.

Nuestro estudio presenta varias fortalezas. La inclusión de radiólogos y patólogos especialistas en el área de estudio y ciegos entre sí, nos permite extraer conclusiones acerca de la capacidad real de la TC para estadificar el CC en nuestro medio. Se utilizó el mismo equipo para todos los exámenes, lo que proporciona imágenes comparables entre sí. Por otro lado, la selección aleatoria de pacientes nos permite disminuir sesgo de selección. Como limitaciones de nuestro trabajo tenemos el carácter unicéntrico del estudio y una mayor proporción de pacientes con CC avanzado que precoz. Sin embargo, para analizar la capacidad de discriminación entre T3 y T4 esta limitación se convierte en fortaleza dado un mayor N para estudio. Si bien los datos nos otorgan una evidencia categórica acerca de la exactitud de la TC en nuestro medio, los datos no son necesariamente generalizables y debieran evaluarse con cautela en otros centros.

Antes de embarcarse en nuevas terapias, es necesario evaluar nuestra capacidad de diagnóstico actual y establecer el nivel de confianza de la infor-
Tabla 5. Estadísticos para discriminar tumores $\mathrm{T} 4$ entre los avanzados (T3-T4)

\begin{tabular}{|lccc|}
\hline & Radiólogo 1 & Radiólogo 2 & Auditado \\
\hline Sensibilidad & 50,0 & 61,1 & 55,6 \\
Especificidad & 85,0 & 75,0 & 85,0 \\
VPP & 75,0 & 68,8 & 76,9 \\
VPN & 65,4 & 68,2 & 68,0 \\
\hline
\end{tabular}

Tabla 6. Estadísticos para diferenciar N (+)

\begin{tabular}{|lccc|}
\hline & Radiólogo 1 & Radiólogo 2 & Auditado \\
\hline Sensibilidad & 60,9 & 69,6 & 60,9 \\
Especificidad & 66,7 & 66,7 & 70,8 \\
VPP & 63,6 & 66,7 & 66,7 \\
VPN & 64,0 & 69,6 & 65,4 \\
\hline
\end{tabular}

mación de nuestros exámenes. Según nuestros resultados, la exactitud de la TC para diferenciar tumores tempranos de avanzados es alta, lo que nos permite identificar a los pacientes en etapas más avanzadas. Sin embargo, es clínicamente relevante poder identificar con certeza los tumores T4, discriminándolos de los tumores T3, porque este sería discutiblemente el factor de mayor relevancia para indicar quimioterapia neoadyuvante en CC. Es justamente aquí donde, según nuestros hallazgos, la exactitud diagnóstica disminuye. Esto último asociado a la baja correlación entre radiólogos expertos para discriminar entre los tumores avanzados, nos sugiere la necesidad de buscar técnicas complementarias para identificar con certeza a los potenciales candidatos a recibir neoadyuvancia.

\section{Responsabilidades éticas}

Protección de personas y animales. Los autores declaran que para esta investigación no se han realizado experimentos en seres humanos ni en animales.

Confidencialidad de los datos. Los autores declaran que en este artículo no aparecen datos de pacientes.

Conflictos de interés: no hay.

Financiamiento: Sin financiamiento externo. 


\section{ARTÍ́CULO ORIGINAL}

\section{Bibliografía}

1. Ferlay J, Ervik M, Lam F, Colombet M, Mery L, Piñeros M, Znaor A, Soerjomataram I BF. Global Cancer Observatory: Cancer Today. [Internet]. Lyon, France: International Agency for Research on Cancer. 2018. Available from: https://gco.iarc.fr/today

2. Bertelsen CA, Neuenschwander AU, Jansen JE, Wilhelmsen M, KirkegaardKlitbo A, Tenma JR, et al. Disease-free survival after complete mesocolic excision compared with conventional colon cancer surgery: a retrospective, population-based study. Lancet Oncol. 2015;16:161-8.

3. Wu C. Systemic Therapy for Colon Cancer. Surg Oncol Clin N Am. 2018;27:235-42.

4. Andre T, Boni C, Navarro M, Tabernero J, Hickish T, Topham C, et al. Improved overall survival with oxaliplatin, fluorouracil, and leucovorin as adjuvant treatment in stage II or III colon cancer in the MOSAIC trial. J Clin Oncol. 2009;27:3109-16

5. Labianca R, Nordlinger B, Beretta GD, Brouquet A, Cervantes A. Primary colon cancer: ESMO Clinical Practice Guidelines for diagnosis, adjuvant treatment and follow-up. Ann Oncol Off
J Eur Soc Med Oncol. 2010;21 Suppl 5:v70-7.

6. Boonstra JJ, Kok TC, Wijnhoven BPL, van Heijl M, van Berge Henegouwen MI, Ten Kate FJW, et al. Chemotherapy followed by surgery versus surgery alone in patients with resectable oesophageal squamous cell carcinoma: Long-term results of a randomized controlled trial. BMC Cancer [Internet]. 2011;11:181. Available from: http://www. biomedcentral.com/1471-2407/11/181

7. Ronellenfitsch U, Schwarzbach M, Hofheinz R, Kienle P, Kieser M, Slanger TE, et al. Perioperative chemo(radio) therapy versus primary surgery for resectable adenocarcinoma of the stomach, gastroesophageal junction, and lower esophagus. Cochrane database Syst Rev. 2013 May;(5):CD008107.

8. Sebag-Montefiore D, Stephens RJ, Steele R, Monson J, Grieve R, Khanna S, et al. Preoperative radiotherapy versus selective postoperative chemoradiotherapy in patients with rectal cancer (MRC CR07 and NCIC-CTG C016): a multicentre, randomised trial. Lancet (London, England). 2009 Mar;373(9666):811-20.

9. Agbamu DA, Day N, Walsh CJ, Hendrickse CW, Langman G, Pallan A, et al. Feasibility of preoperative chemotherapy for locally advanced, operable colon cancer: The pilot phase of a randomised controlled trial. Lancet Oncol [Internet]. 2012;13(11):1152-60. Available from: http://dx.doi.org/10.1016/ S1470-2045(12)70348-0.

10. Nerad E, Lahaye MJ, Maas M, Nelemans P, Bakers FCH, Beets GL, et al. Diagnostic Accuracy of CT for Local Staging of Colon Cancer: A Systematic Review and Meta-Analysis. AJR Am J Roentgenol. 2016;207:984-95.

11. Weiser MR. AJCC 8th Edition: Colorectal Cancer. Vol. 25, Annals of Surgical Oncology. United States; 2018. p. 1454-5.

12. Olsen ASF, Gundestrup AK, Kleif J, Thanon T, Bertelsen CA. Accuracy of preoperative staging with multidetector computed tomography in colon cancer. Colorectal Dis. 2021;23:680-8. doi: 10.1111/codi.15415. Epub 2020 Dec 26

13. Korsbakke K, Dahlbäck C, Karlsson N, Zackrisson S, Buchwald P. Tumor and nodal staging of colon cancer: accuracy of preoperative computed tomography at a Swedish high-volume center. Acta Radiol Open 2019 Dec 30;8(12):2058460119888713. doi: 10.1177/2058460119888713. eCollection 2019 Dec. 